\title{
Low-mass, helium-enriched PG 1159 stars: a possible evolutionary origin and implications for their pulsational stability properties
}

\author{
L. G. Althaus ${ }^{1,2, \star}$, A. H. Córsico ${ }^{1,2}$, and M. M. Miller Bertolami ${ }^{1,2, \star \star}$ \\ 1 Facultad de Ciencias Astronómicas y Geofísicas, Universidad Nacional de La Plata, Paseo del Bosque S/N, (B1900FWA) La Plata, \\ Argentina \\ 2 Instituto de Astrofísica La Plata, IALP, CONICET-UNLP \\ e-mail: [althaus, acorsico,mmiller]@fcaglp.unlp.edu.ar
}

Received 6 December 2006 / Accepted 21 February 2007

\begin{abstract}
Aims. We examine a recently-proposed evolutionary scenario that could explain the existence of the low-mass, helium-enriched PG 1159 stars. We focus in particular on studying the pulsational stability properties of the evolutionary models predicted by such a scenario.

Methods. We assess the overstability of pulsation $g$-modes of stellar models as evolution proceeds in the PG 1159 domain. Stellar models are extracted from the full evolution of a $1-M_{\odot}$ model star that experiences its first thermal pulse as a late thermal pulse (LTP) after leaving the AGB. The evolutionary stages corresponding to the born-again episode and the subsequent helium sub-flashes are taken into account in detail.

Results. Under reasonable assumptions of mass-loss rate, the evolutionary scenario reproduces the high helium abundances observed in some PG 1159 stars. We find that, despite the high helium abundance in the driving layers, a narrow region exists in the $\log T_{\text {eff }}-\log g$ diagram for which the helium-enriched PG 1159 sequence exhibits unstable pulsation modes with periods in the range 500 to $1600 \mathrm{~s}$. In particular, the nonpulsating helium-enriched PG 1159 star, MCT 0130-1937, is located outside the theoretical instability domain. Our results suggest that MCT 0130-1937 is a real non-pulsating star and that the lack of pulsations should not be attributed to unfavorable geometry.

Conclusions. Our study hints at a consistent picture between the evolutionary scenario that could explain the existence of heliumenriched PG 1159 stars and the nonvariable nature of MCT 0130-1937. We also present theoretical support for the unusually high helium abundance observed in the nonpulsating PG 1159 star HS 1517+7403. We suggest that HS 1517+7403 could be a transition object linking the low-mass helium-rich $\mathrm{O}(\mathrm{He})$ stars with the helium-enriched PG 1159 stars via the evolutionary connection K1-27 $\rightarrow$ HS 1517+7403 $\rightarrow$ MCT 0130-1937.
\end{abstract}

Key words. stars: evolution - stars: abundances - stars: AGB and post-AGB - stars: interiors - stars: variables: general white dwarfs

\section{Introduction}

PG 1159 stars constitute the evolutionary link between the asymptotic giant branch (AGB) stars and most of the hydrogendeficient white dwarf (WD) stars. Currently, 37 stars are members of the PG 1159 family, which span a wide domain in the $\log T_{\text {eff }}-\log g$ diagram ( $g$ in cgs units): $5.5 \lesssim \log g \lesssim 8$ and $75000 \mathrm{~K} \lesssim T_{\text {eff }} \lesssim 200000 \mathrm{~K}$; see Werner \& Herwig (2006) for a review. These hot stars are thought to be formed via a bornagain episode resulting either from a very late thermal pulse (VLTP) experienced by a hot WD during its early cooling phase - see Schönberner (1979) and Iben et al. (1983) for earlier references - or a late thermal pulse (LTP) that occurs during the post-AGB evolution when hydrogen burning is still active; see Blöcker (2001) for references. During the VLTP, the convection zone driven by the helium flash reaches the hydrogen-rich envelope of the star, with the consequence that most of the hydrogen content is burnt. The star is then forced to evolve rapidly back to the AGB and finally into the domain of the PG 1159 stars at high

* Member of the Carrera del Investigador Científico y Tecnológico, CONICET, Argentina.

$\star \star$ Fellow of CONICET, Argentina

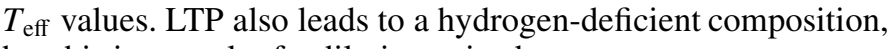
but this is a result of a dilution episode.

Interest in PG 1159 stars is likewise motivated by the fact that eleven of them exhibit multiperiodic luminosity variations induced by nonradial $g$-mode pulsations. Pulsating PG $1159-$ commonly referred to as GW Virginis variables - show lowdegree $(\ell \leq 2)$, high-radial order $(k \gtrsim 18) g$-modes with periods in the range from about 300 to $3000 \mathrm{~s}$. About half of them are still surrounded by a planetary nebula. Since the pioneering works of Starrfield et al. (1983, 1984) - see also Starrfield et al. (1985) and Stanghellini et al. (1991) - the pulsation driving mechanism and, in particular, the chemical composition of the driving zone in PG 1159 stars have been the subject of hot debate. The works of Saio (1996) and Gautschy (1997) and, more recently, Quirion et al. (2004), Gautschy et al. (2005), and Córsico et al. (2006) have convincingly demonstrated that $g$-mode pulsations in the range of the periods of GW Virginis stars can be easily driven in PG 1159 models with a uniform envelope composition - compatible with observed photospheric abundances - through the $\kappa$-mechanism associated with the cyclical partial ionization of the K-shell electrons of carbon and/or oxygen in the envelope of these models. 
A distinctive feature characterizing PG 1159 stars is their peculiar surface chemical composition. Indeed, PG 1159 stars exhibit surface layers dominated by helium, carbon, and oxygen, the main nucleosynthetic constituents of the previous intershell layer of their AGB progenitor stars. Typically, surface abundances $^{1}$ of about $0.33 \mathrm{He}, 0.5 \mathrm{C}$, and $0.17 \mathrm{O}$ are reported, with notable variations from star to star (Dreizler \& Heber 1998; Werner 2001). In fact, almost every star has its individual $\mathrm{He} / \mathrm{C} / \mathrm{O}$ mixture. As reported by Werner (2001) and Werner \& Herwig (2006), the helium abundance ranges between $0.30-0.85$, and carbon and oxygen abundances span the ranges $0.15-0.60$ and $0.02-0.20$, respectively. In particular, the helium abundance mostly covers the range $0.3-0.5$; only a minority of PG 1159 stars show a helium-enriched abundance in the range $0.6-0.85$.

The dispersion in the atmospheric composition of PG 1159 stars is relevant to the question of the excitation of pulsation modes in models of these stars and, particularly, of the coexistence of variable and non-variable stars in the domain of the GW Virginis, a longstanding issue recently addressed by Quirion et al. (2004). The helium-enriched and nitrogen-deficient nonpulsating PG 1159 star MCT 0130-1937 is particularly outstanding. In fact, this object - characterized by a surface composition of about $0.74 \mathrm{He}, 0.22 \mathrm{C}$, and $0.03 \mathrm{O}$ (Werner \& Herwig 2006) and a stellar mass of $0.54 M_{\odot}$ (Miller Bertolami \& Althaus 2006) - is well inside the PG 1159 instability domain in the $\log T_{\text {eff }}-\log g$ diagram (Córsico et al. 2006). Even more remarkable is the fact that, in the $\log T_{\text {eff }}-\log g$ diagram, this star is located between two pulsating stars, PG 2131+066 and PG $1707+427$, where both of them have standard helium abundances (0.44). Other relevant examples of nonpulsating PG 1159 stars within the instability domain are the low-mass stars HS $0704+6153$ with a surface helium abundance of 0.69 and HS $1517+7403$ with an unusually high helium abundance of 0.85 .

With the help of static PG 1159 models, Quirion et al. (2004) find that the high helium abundance should be the cause of the absence of pulsations in MCT 0130-1937. Indeed, the envelope of MCT 0130-1937 is so helium-enriched compared to its two neighboring GW Virginis stars that the "helium poisoning" would be responsible for the lack of pulsations in this star. However, it should be noted that the non detection of pulsations in this object could be due to unfavorable geometry. For instance if the star is observed pole-on, modes with $m= \pm \ell$ will not be detected.

The wide variety of surface patterns observed in PG 1159 stars poses a major challenge to the theory of post-AGB evolution. Although the dispersion in the surface composition observed in PG 1159 stars can admittedly be understood in terms of the standard born-again AGB star scenario ${ }^{2}$ (Herwig et al. 1999; Miller Bertolami \& Althaus 2006), the existence of helium-enriched and nitrogen-deficient objects such as MCT 0130-1937 is certainly more difficult to explain, and it still deserves investigation. This is the precise focus of the present work. Specifically, we examine the evolutionary scenario for the formation of low-mass, helium-enriched PG 1159 stars as recently proposed by Miller Bertolami \& Althaus (2006) by assessing the overstability of pulsation $g$-modes of the resulting stellar models as evolution proceeds in the PG 1159

\footnotetext{
1 Throughout this paper, we shall use abundances by mass fraction.

2 The spread in surface composition of most PG 1159 stars can be explained by differences in stellar mass and the number of thermal pulses experienced by progenitors during the AGB.
}

domain. As shown in Miller Bertolami \& Althaus (2006), this scenario could provide a possible explanation for the existence of PG 1159 stars, such as the above-mentioned HS 0704+6153, HS 1517+7403, and MCT 0130-1937, three helium-enriched and nitrogen-deficient objects in the sample of Dreizler \& Heber (1998). The following section contains details about the main aspects of this evolutionary scenario and the stellar models we used as a background for the pulsation stability analysis described in Sect. 3. Our aim is to assess to what extent the pertinent sequence of stellar models do not show any sign of variability in the domain of the GW Virginis stars, thus having a consistent picture of the evolutionary and pulsational status of MCT 0130-1937. Finally, Sect. 4 is devoted to discussing and summarizing our results. In particular, we discuss there the evolutionary connection $\mathrm{K} 1-27 \rightarrow \mathrm{HS} 1517+7403 \rightarrow$ MCT 0130-1937 that would link the helium-rich $\mathrm{O}(\mathrm{He})$ stars with the helium-enriched PG 1159 stars and would explain the unusually high helium abundance observed in HS 1517+7403.

\section{Evolutionary scenario}

The evolutionary scenario proposed by Miller Bertolami \& Althaus (2006) involves computating the advanced stages of the evolution of a low-mass star that, as a result of mass-loss events, avoids the thermally pulsing AGB phase, and it experiences its first thermal pulse as an LTP after leaving the AGB. Indeed, helium abundances as high as observed in the helium-enriched PG 1159 stars are typical of the intershell helium abundances that develops during the first thermal pulse $\left(\sim 0.75^{3}\right)$. Because of the small envelope mass characterizing low-mass stars, this scenario appears to be quite likely in these stars.

The Hertzsprung-Russell diagram corresponding to the evolutionary scenario is shown in Fig. 1. Artificial mass-loss rates force the initially $1-M_{\odot}$ model star to undergo its first thermal pulse as an LTP at about $10000 \mathrm{~K}$, after leaving the AGB. After the short-lived born-again episode and before the PG 1159 domain, the remnant experiences two additional excursions to lower temperatures - the two double-loop paths in Fig. 1 - by virtue of helium sub-flashes. The total time spent by the remnant in the red during these loop episodes amounts to about 3000 yr. During this time, mass-loss episodes are expected to erode the outer envelope considerably. For instance, mass-loss rates as high as $10^{-5} M_{\odot} / \mathrm{yr}$ and even higher have been observed in Sakurai's object (Hajduk et al. 2005).

In the interest of helping to understand the evolutionary connections to be studied later, we show the chemical stratification by the end of the born-again stage in Fig. 2 (about $300 \mathrm{yr}$ after the occurrence of the LTP). This figure illustrates the inner ${ }^{1} \mathrm{H},{ }^{4} \mathrm{He}$, ${ }^{12} \mathrm{C},{ }^{14} \mathrm{~N}$, and ${ }^{16} \mathrm{O}$ distribution in terms of the outer mass fraction $q$, where $q=1-m_{r} / M_{*}$. Note the presence of the intershell layer below the thin helium buffer, which results from the short-lived mixing episode during the helium flash at the LTP. This intershell layer of $0.04 M_{\odot}$ is substantially enriched in helium and deficient in nitrogen: $\left[{ }^{4} \mathrm{He},{ }^{12} \mathrm{C},{ }^{16} \mathrm{O}\right]=[0.73,0.21,0.03]$. Despite the small envelope $\left(3.7 \times 10^{-3} M_{\odot}\right)$ overlying the intershell region, no strong dredge-up occurs. This is expected because of the low intensity of the first thermal pulse in low-mass stars. By assuming mass-loss rates within observational expectations, Miller Bertolami \& Althaus (2006) found that the hydrogen-rich outer envelope is eroded and surface abundances start to change

\footnotetext{
3 During subsequent thermal pulses, the helium abundance in the intershell layer gradually decreases if convective extramixing like overshooting is considered; see Herwig (2000).
} 


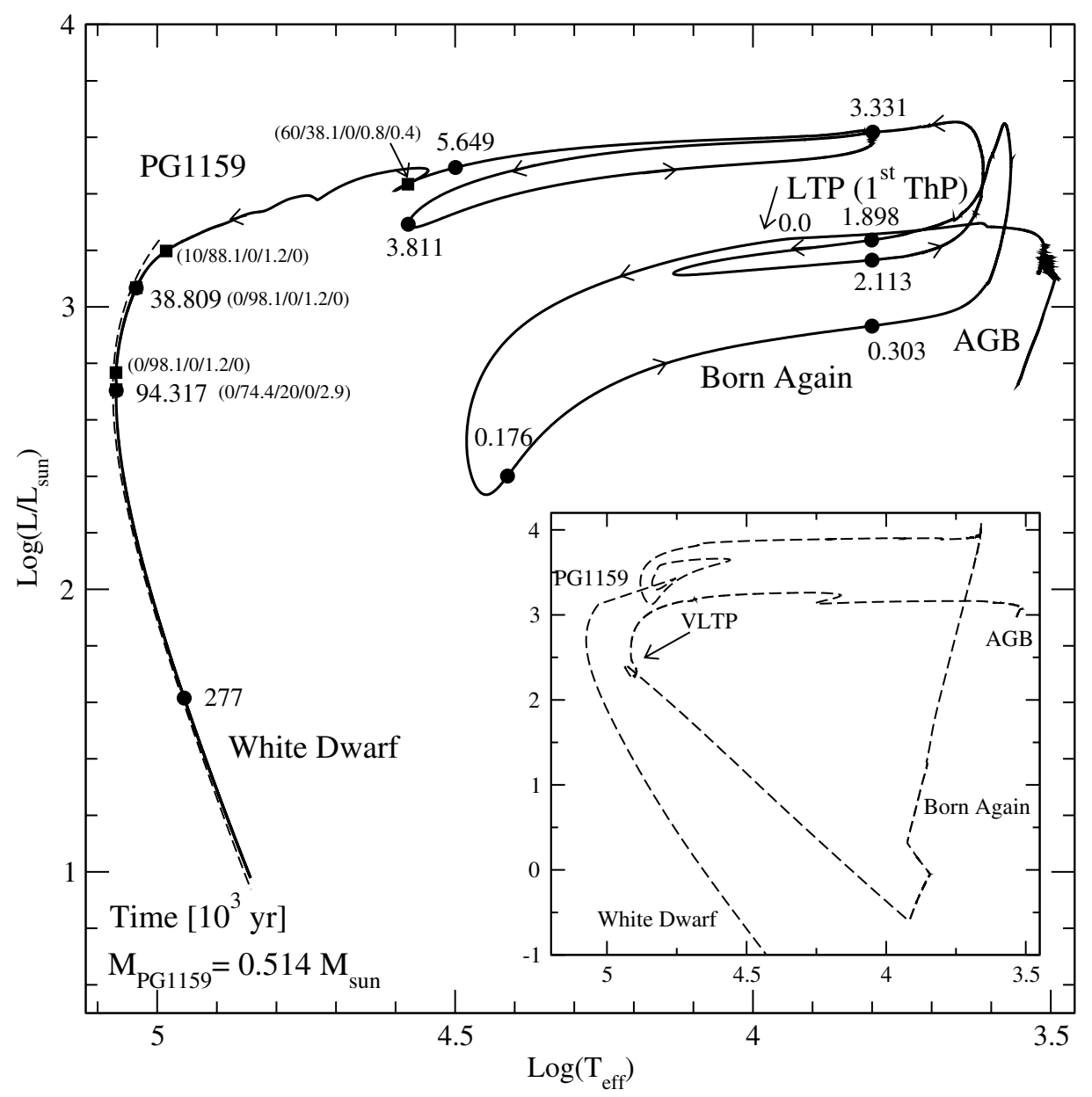

Fig. 1. Hertzsprung-Russell diagram for the evolutionary scenario that leads to the formation of helium-enriched PG 1159 stars. The star experiences its first thermal pulse as an LTP. Numbers beside filled circles and squares along the track give, respectively, the age (in $10^{3} \mathrm{yr}$ ) counted from the occurrence of the LTP and the surface abundances $\left({ }^{1} \mathrm{H} /{ }^{4} \mathrm{He} /{ }^{12} \mathrm{C} /{ }^{14} \mathrm{~N} /{ }^{16} \mathrm{O}\right)$ in percent. The evolutionary track resulting from a VLTP episode is also shown with dashed lines for the PG 1159 regime; the complete track is depicted in the inset.

gradually by $\log T_{\text {eff }} \sim 4.6$, well before the sequence reaches the domain of the PG 1159 stars.

From this point until the intershell chemistry is uncovered, the nitrogen surface abundance remains 0.012 with no trace of carbon. Thirty thousand yr later - at $T_{\text {eff }} \sim 108000 \mathrm{~K}-$ the last vestiges of hydrogen-rich material left in the star are removed and the surface exhibits the buffer abundances: $\left[{ }^{4} \mathrm{He},{ }^{12} \mathrm{C},{ }^{14} \mathrm{~N}\right.$, $\left.{ }^{16} \mathrm{O}\right]=[0.98,0,0.012,0]$. These will be the surface abundances until the helium buffer is eroded by further mass loss during the subsequent $50000 \mathrm{yr}$ of evolution (see Fig. 1). During this time, the surface abundance will be typical of those exhibited by $\mathrm{O}(\mathrm{He})$ stars. Finally, when the helium buffer is removed, the models diplay the intershell abundances $-\left[{ }^{4} \mathrm{He},{ }^{12} \mathrm{C},{ }^{14} \mathrm{~N}\right.$, $\left.{ }^{16} \mathrm{O}\right]=[0.74,0.20,0,0.029]-$ characteristic of the heliumenriched PG 1159 stars.

The location of these abundance changes in the $\log T_{\text {eff }}-$ $\log g$ diagram is shown in Fig. 3. We also depict the location of two helium-rich $\mathrm{O}(\mathrm{He})$ stars: K1-27 and HS 2209+8229. In particular K1-27, for which detailed abundances have been derived, displays an almost pure helium composition (0.98) with traces of ${ }^{14} \mathrm{~N}(0.017)$ - however, small hydrogen and carbon contents cannot be excluded, see Rauch et al. (1998) - which are similar to the surface abundances predicted by the evolutionary scenario by the time the small helium buffer is uncovered: $0.98{ }^{4} \mathrm{He}$ and $0.012{ }^{14} \mathrm{~N}$. In addition, we include in this figure the three helium-enriched and nitrogen-deficient PG 1159 stars from the sample of the non-pulsating stars of Dreizler \& Heber (1998): HS 0704+6153, HS 1517+7403, and MCT 0130-1937 with abundances of $\mathrm{He} / \mathrm{C} / \mathrm{O}=0.69 / 0.21 / 0.09,0.84 / 0.13 / 0.02$, and $0.74 / 0.22 / 0.03$, respectively. Note that the evolutionary sequence nearly reproduces both the location in the $\log T_{\text {eff }}-\log g$ diagram and the surface composition of the three heliumenriched PG 1159 stars (in particular MCT 0130-1937) and the $\mathrm{O}(\mathrm{He})$ star $\mathrm{K} 1-27$. It is apparent from Fig. 3 that this scenario suggests an evolutionary connection between the $\mathrm{O}(\mathrm{He})$ stars with low mass (namely K1-27 and HS 2209+8229) and the helium-enriched PG 1159 stars. Note from Fig. 3 that K1-27 is consistent with a stellar mass value of $M<0.514 M_{\odot}$. This is markedly lower than the value quoted by Rauch et al. (1998) of $0.55 M_{\odot}$. The difference can be traced back to the fact that the Rauch et al. (1998) determination is based on the Blöcker (1995) hydrogen-burning evolutionary tracks, while our determination is based on evolutionary sequences with surface abundances consistent with those observed in this $\operatorname{star}^{4}$. The same trend is also present in the new mass determinations of PG 1159 stars given in Miller Bertolami \& Althaus (2006), as compared with the mass values quoted by Werner \& Herwig (2006), based also on hydrogen-burning post-AGB tracks.

\footnotetext{
${ }^{4}$ We are performing new simulations that show that tracks for $\mathrm{H}$ and He burning post-AGB stars are remarkably different for low-mass remnants $\left(M<0.55 M_{\odot}\right)$.
} 


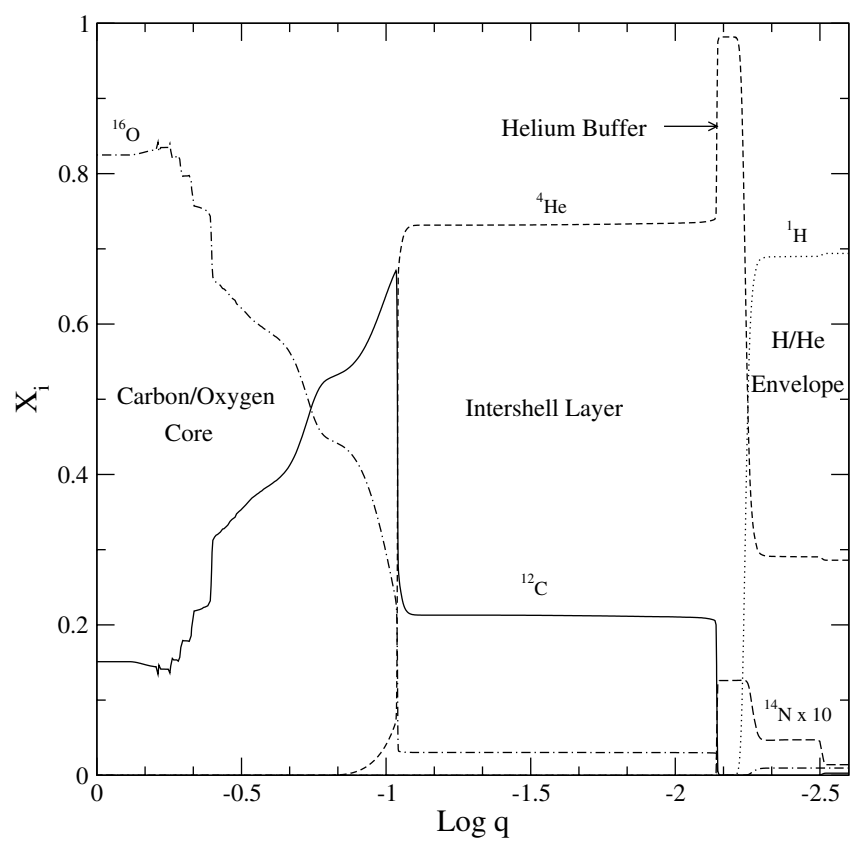

Fig. 2. Internal abundance distribution of ${ }^{1} \mathrm{H},{ }^{4} \mathrm{He},{ }^{12} \mathrm{C},{ }^{14} \mathrm{~N}$, and ${ }^{16} \mathrm{O}$ as a function of the outer mass fraction $q$ for a model at $300 \mathrm{yr}$ after the occurrence of the LTP (see Fig. 1). The location of the helium buffer and the underlying intershell layer are indicated.

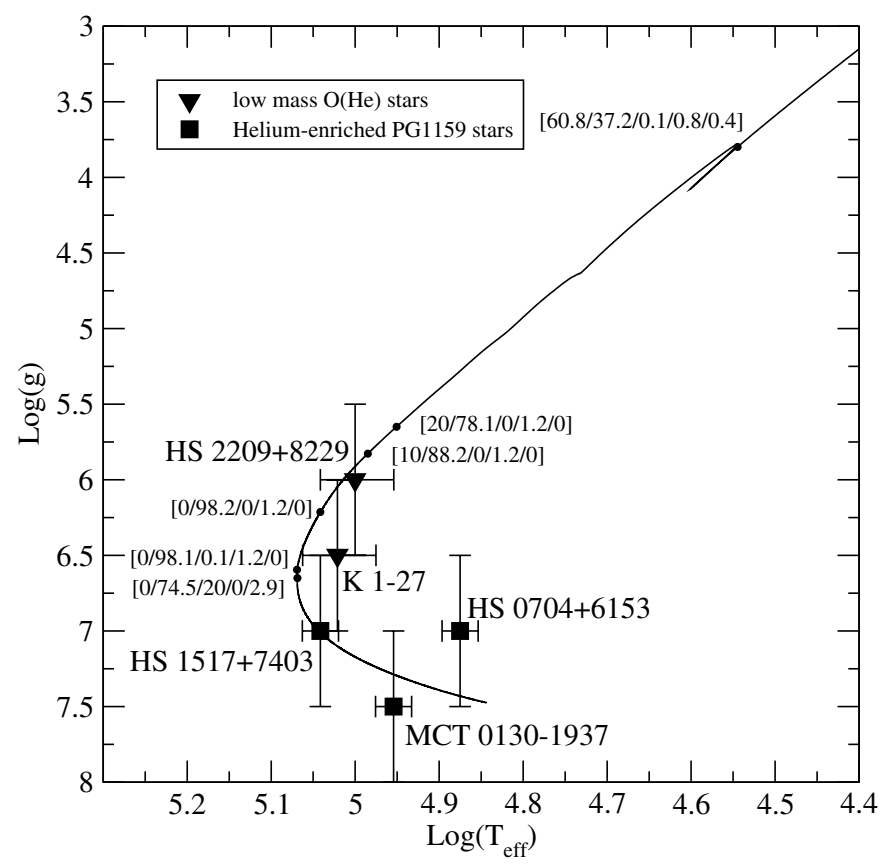

Fig. 3. The $\log T_{\text {eff }}-\log g$ evolution of the $0.514 M_{\odot}$ remnant. Surface abundances $\left[{ }^{1} \mathrm{H} /{ }^{4} \mathrm{He} /{ }^{12} \mathrm{C} /{ }^{14} \mathrm{~N} /{ }^{16} \mathrm{O}\right]$ are given at selected stages (black dots). We also show the observational predictions for two low-mass $\mathrm{O}(\mathrm{He})$ stars and three helium-enriched PG 1159 stars (triangles and square symbols, respectively) taken from Rauch et al. (1998) and Dreizler \& Heber (1998).

A relevant issue given by the evolutionary scenario concerns the age predicted for K1-27. Specifically, we find that our sequence predicts an age of about 30-60 Kyr at the observed effective temperature and surface composition of K1-27. This age is a factor of 5-3 smaller than the age quoted by Rauch et al. (1994), strongly alleviating the discrepancy between the evolutionary age and the expansion age of the nebula ${ }^{5}$. This fact makes the plausibility of the evolutionary scenario for the origin of this star even more attractive.

Needless to say, the exact location in the $\log T_{\text {eff }}-\log g$ diagram where the star will show $\mathrm{O}(\mathrm{He})$ - or helium-enriched surface abundances depends on the actual course of mass-loss events. To assess this, we re-computed the post VLT evolution of the sequence but for the case in which mass-loss rates are one order of magnitude lower than assumed in Miller Bertolami \& Althaus (2006). In particular, we assumed a mass-loss rate during the giant state of only $10^{-7} M_{\odot} / \mathrm{yr}$. As a result, the surface layers of the models stay hydrogen-and helium-rich throughout the evolution. This places a lower limit - below observational expectations - on the mass-loss rates for which the evolutionary scenario may explain the existence of helium-enriched PG 1159 stars like MCT 0130-1937.

Finally, we note that the low-mass, helium-enriched PG 1159 stars could be the result of a VLTP instead an LTP. To assess this we forced our post-AGB sequence to undergo a VLTP episode. In Fig. 1 we include the resulting track. The full track is shown in the inset. Note that for the PG 1159 regime, both tracks are almost indistinguishable. As in the of case for the LTP, the surface chemistry of the emerging star will essentially be that of the intershell layer $\left[{ }^{4} \mathrm{He},{ }^{12} \mathrm{C},{ }^{16} \mathrm{O}\right]=[0.74,0.16,0.03]$, but - because of the burning of the hydrogen content - enriched in ${ }^{13} \mathrm{C}$ and nitrogen (0.01). The presence of nitrogen is at odds with observations in the low-mass, helium-enriched PG 1159 stars. This fact discards the possibilty that the progenitors of these stars have experienced a VLTP episode. Note also that because the entire helium-rich buffer is engulfed by the helium flash convection zone during the VLTP, the development of a helium-rich surface composition characteristic of the $\mathrm{O}(\mathrm{He})$ stars is certainly not expected in this case. In addition, we want to comment on the fact that, from our numerical experiments, the occurrence of a VLTP episode in very low-mass stars $\left(M \lesssim 0.53 M_{\odot}\right)$ appears to require a delicate fine tuning of the mass-loss rate. Thus, we are tempted to conclude that in these stars, VLTP episodes might be less likely than the LTP ones.

\section{Pulsational stability properties}

The evolutionary scenario proposed in Miller Bertolami \& Althaus (2006) provides a possible explanation for the existence of helium-enriched PG 1159 stars like MCT 0130-1937, linking them with the low-mass $\mathrm{O}(\mathrm{He})$ stars. In the frame of recent stability calculations (Quirion et al. 2004), we should expect that - as a result of helium poisoning - the pertinent stellar configurations do not show any sign of variability in the domain of the pulsating PG 1159 stars. To assess this, we performed pulsation stability analysis on our models. It is important to note that we are examining the stability properties of stellar models belonging to a real evolutionary sequence derived from the complete history of the progenitor star, an aspect that renders robustness to our pulsational results.

We performed a pulsation $g$-mode stability analysis by employing the linear, nonradial, nonadiabatic pulsation code described in Córsico et al. (2006), which has recently been employed to reexamine the theoretical instability domain of pulsating PG 1159 stars. This code assumes the "frozen-in convection" approximation in which the perturbation of the convective luminosity is ignored.

\footnotetext{
${ }^{5}$ We note that the kinematical age of the nebula is not expected to change appreciably as a result of our lower stellar mass for K1-27.
} 


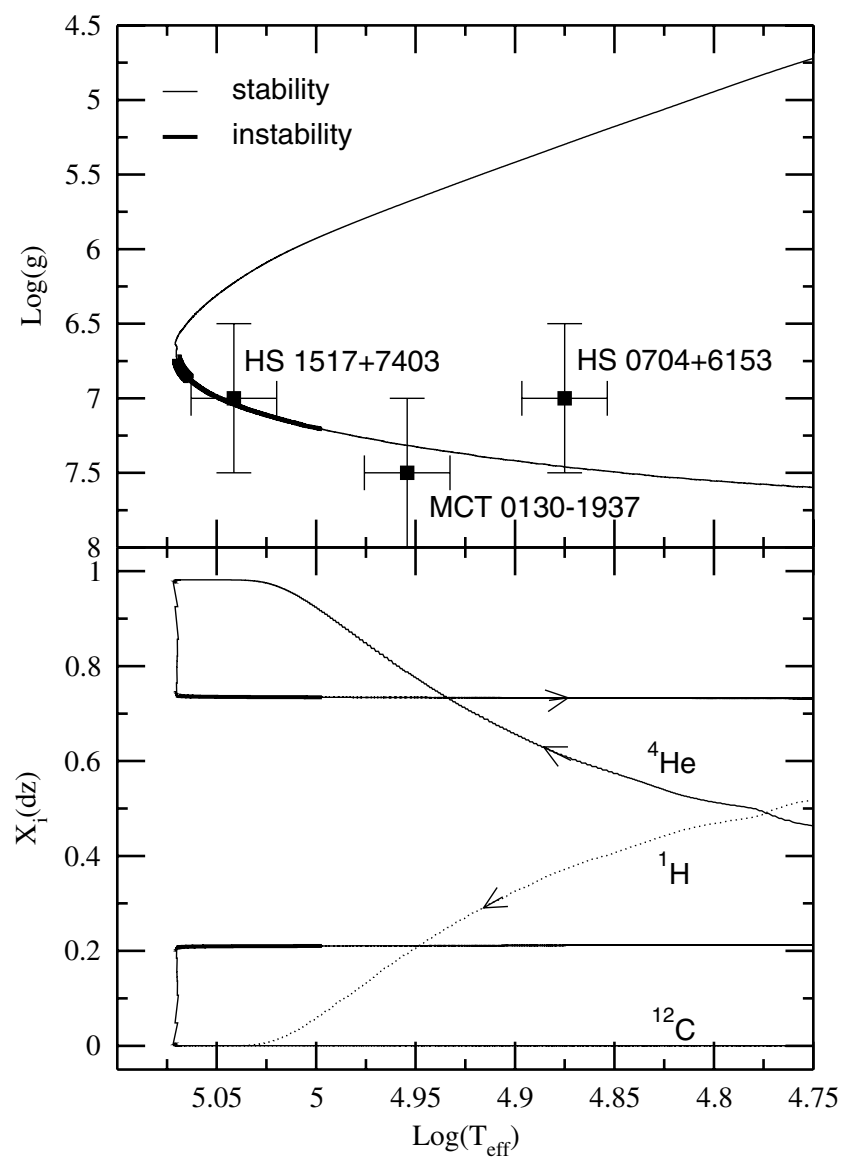

Fig. 4. Upper panel: $\log T_{\text {eff }}-\log g$ evolution of our $0.514-M_{\odot}$ remnant and the location of the three helium-enriched PG 1159 stars according to Dreizler \& Heber (1998): HS 1517+7403, HS 0704+6153, and MCT 0130-1937. The thick portion of the curve corresponds to the instability domain of our sequence, and the thickest portion near the knee of the track corresponds to the case for which the helium abundance of our model sequence is artificially changed to 0.85 (see Sect. 4). Bottom panel: Chemical abundances at the driving region $(\log T \sim 6.2)$ in terms of the effective temperature. Arrows indicate the course of evolution.

We analyzed the stability properties of stellar models covering a range of $5.1 \gtrsim \log \left(T_{\text {eff }}\right) \gtrsim 4.7$. For each model we restricted our study to $\widetilde{\ell}=1 \mathrm{~g}$-modes with periods in the range $50 \mathrm{~s} \lesssim \Pi \lesssim 5000 \mathrm{~s}$, thus comfortably embracing the full period spectrum observed in pulsating PG 1159 stars. Surprisingly enough, we find that, despite the high helium abundance in the driving layers, there is a region in the $\log T_{\text {eff }}-\log g$ diagram for which our helium-enriched PG 1159 sequence exhibits unstable pulsation modes. These modes are driven by the $\kappa$-mechanism associated with the opacity bump due to partial ionization of carbon and oxygen; see Gautschy et al. (2005) and Córsico et al. (2006). To illustrate this, we present two panels in Fig. 4 that display the $\log T_{\text {eff }}-\log g$ plane (upper panel) and the chemical abundances at the driving region (bottom panel) in terms of the effective temperature. Note the presence of a well-defined, though short, instability domain on the $\log T_{\text {eff }}-\log g$ plane (thick portion of the track). It is clear that the extension of the instability domain is markedly dependent on the helium abundance in the outer layers. Indeed, it is only by the time mass loss has eroded the helium buffer and exposed the helium-and carbon-rich intershell layer - bottom panel in Fig. 4 - that our models start to exhibit pulsational instability. From the modal diagram in Fig. 5, this instability starts to manifest itself at the

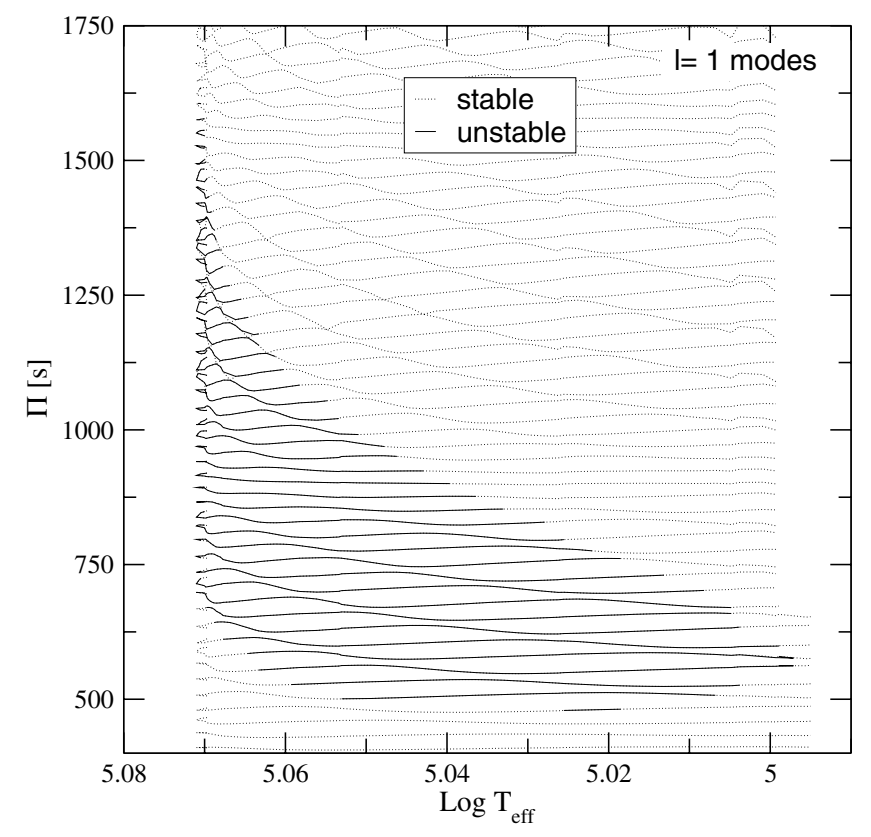

Fig. 5. Dipole modal diagram (periods vs. effective temperature) for our helium-enriched evolutionary sequence. Solid (dotted) lines denote pulsational instability (stability). Unstable mode periods range from about 500 to $1600 \mathrm{~s}$. Below $T_{\text {eff }} \sim 100000 \mathrm{~K}$, no unstable pulsation modes are found.

longest pulsation periods. Note that unstable mode periods range from about 500 to $1600 \mathrm{~s}$. Also note the strong decrease in the longest expected periods; this is because the high helium content in the driving region. By the time evolution has proceeded to $T_{\text {eff }} \sim 100000 \mathrm{~K}$ our models can no longer drive unstable modes and they become stable with further evolution (see Fig. 5). This behavior is in sharp contrast to the situation encountered in lowmass PG 1159 models with standard surface helium abundances, which show pulsational instability throughout this region of the $\log T_{\text {eff }}-\log g$ diagram (Córsico et al. 2006).

It is clear from Fig. 4 that the nonpulsating MCT 0130-1937 star is located well outside the theoretical instability domain. In fact, we do not find any sign of instability in our models by the time evolution has reached the domain of MCT 0130-1937. This result hints at a consistent picture between the evolutionary scenario described in Sect. 2 that could explain the existence of helium-enriched PG 1159 stars and the nonvariable nature of MCT 0130-1937.

\section{Discussion and conclusions}

We have examined the evolutionary scenario proposed by Miller Bertolami \& Althaus (2006), which could explain the existence of low-mass, helium-enriched PG 1159 stars. In particular, this scenario remarkably reproduces both the location in the $\log T_{\text {eff }}-\log g$ diagram and the helium-enriched and nitrogendeficient composition of MCT 0130-1937, and also suggests a possible evolutionary connection between the low-mass, heliumrich $\mathrm{O}(\mathrm{He})$ stars (namely $\mathrm{K} 1-27$ and HS 2209+8229) and the helium-enriched PG 1159 stars.

In this paper we have assessed the overstability of $g$-modes of the pertinent stellar models as evolution proceeds in the PG 1159 domain. We find that - despite the high helium abundance in the driving layers - there exists a region in the $\log T_{\text {eff }}-\log g$ diagram for which our helium-enriched PG 1159 sequence exhibits unstable pulsation modes with periods in the 
range 500 to $1600 \mathrm{~s}$. The domain of instability is restricted to a rather narrow region of the $\log T_{\text {eff }}-\log g$ diagram. In particular, MCT 0130-1937 is located outside the theoretical instability domain. In this sense, this finding reinforces the conclusions arrived at in Quirion et al. (2004) about the nonvariability of MCT 0130-1937. This result hints at a consistent picture between the evolutionary scenario proposed by Miller Bertolami $\&$ Althaus (2006) for the origin of low-mass, helium-enriched PG 1159 stars and the nonvariable nature of MCT 0130-1937. We conclude that MCT 0130-1937 is probably a real nonpulsating star and that the lack of pulsations cannot be attributed to unfavorable geometry.

However, as documented in Fig. 4, the nonpulsating heliumenriched object HS 1517+7403 lies well inside the predicted instability domain of our sequence. The presence of this nonvariable star in the unstable region of our sequence could be understood in terms of its higher surface helium abundance (about 0.85, see Werner \& Herwig 2006) as compared with that of MCT 0130-1937. In fact, the helium surface abundance of HS $1517+7403$ is intermediate between the intershell helium abundance $(0.75)$ of our models and that of the helium buffer (0.98), for which no unstable modes are found at all. For a quantitative inference, we have recomputed the post born-again evolution of our sequence by artificially changing the surface abundance to be compatible with that of HS 1517+7403. Our pulsational analysis shows in this case that, though a very narrow instability domain still persists - see Fig. 4 - HS 1517+7403 is outside the resulting theoretical unstable region. A helium abundance as high as observed in HS $1517+7403$ appears to be required to starve pulsations in this star. In contrast, we saw that unstable modes are excited for a helium abundance of 0.75 . This theoretical finding strongly supports the observational expectation for the unusually high helium abundance in HS 1517+7403.

From an evolutionary point of view, the high helium abundance in HS $1517+7403$ is difficult to understand. Indeed, helium abundances larger than about 0.75 are not expected in the intershell layer during the AGB evolution. In addition, the presence of abundant carbon at its surface - about 0.13 by mass, Werner \& Herwig (2006 - reflects the occurrence of helium burning in prior evolutionary stages. It is conceivable that the surface composition observed in HS $1517+7403$ could be reflecting the chemical abundance distribution existing in the narrow transition layer between the helium buffer and the massive intershell region - see Fig. 2. This layer comprises only about $6.5 \times 10^{-5} M_{\odot}$. With our adopted mass-loss rates, this layer is rapidly eroded in a matter of $6500 \mathrm{yr}$. During this time our sequence barely evolves in the $\log T_{\text {eff }}-\log g$ diagram see Fig. 3. But for similar mass-loss rates to those charaterizing $\mathrm{O}(\mathrm{He})$ stars (about $10^{-9} M_{\odot} / \mathrm{yr}$ ) - a strong reduction in the mass-loss rate is indeed expected with decreasing luminosity - this layer would be eroded slow enough for the remnant to evolve to the domain of HS $1517+7403$ with surface abundances similar to those observed in this star. This prompts us to suggest that HS $1517+7403$ could be a transition object between the low-mass $\mathrm{O}(\mathrm{He})$ stars and the helium-enriched PG 1159 stars like MCT 0130-1937.

The evolutionary scenario proposed by Miller Bertolami \& Althaus (2006) suggests the possibility that low-mass $\mathrm{O}(\mathrm{He})$ stars could be the direct progenitors of the helium-enriched PG 1159 stars, and not form a distinct post-AGB evolutionary channel, for instance, the result of a stellar merging event. The plausibility of this scenario is sustained not only by spectroscopic evidence but also, as shown in this work, by consistent pulsational stability calculations of helium-enriched PG 1159 models. In addition, the substantially younger ages predicted by this scenario for $\mathrm{K} 1-27$ are in line with the expected kinematical age of the nebula. Finally, we have put forward the possibility of an evolutionary connection $\mathrm{K} 1-27 \rightarrow \mathrm{HS} 1517+7403$ $\rightarrow$ MCT 0130-1937. The existence of these evolutionary links appears more attractive in view of the observational fact that the helium-enriched PG 1159 stars are nitrogen-deficient, which rules out the occurrence of a VLTP episode during the progenitor evolution. In case a VLTP had occurred, then an homogeneous composition - corresponding to the intershell chemistry - throughout the envelope would have been expected immediately after the born again episode, with the result that no marked surface abundance change would have resulted from mass-loss episodes during the later evolution. Finally, detailed tabulations of the calculations presented here are available at our web site: http://www. fcaglp. unlp. edu . ar/evolgroup/.

Acknowledgements. We warmly acknowledge the comments and suggestions of our referee, which have improved both the contents and presentation of the paper. This research was supported by the Instituto de Astrofísica La Plata and by the PIP 6521 grant from CONICET. We thank H. Viturro and R. Martinez for technical support.

\section{References}

Blöcker, T. 1995, A\&A, 299, 755

Blöcker, T. 2001, ApSS, 275, 1

Córsico, A. H., Althaus, L. G., \& Miller Bertolami, M. M. 2006, A\&A, 458, 259 Dreizler, S., \& Heber, U. 1998, A\&A, 334, 618

Gautschy, A. 1997, A\&A, 320, 811

Gautschy, A., Althaus, L. G., \& Saio, H. 2005, A\&A, 438, 1013

Hajduk, M., Zijlstra, A. A., Herwig, F., et al. 2005, Science, 308, 231

Herwig, F. 2000, A\&A, 360, 952

Herwig, F., Blöcker, T., Langer, N., \& Driebe, T. 1999, A\&A, 349, L5

Iben, I. Jr., Kaler, J. B., Truran, J. W., \& Renzini, A. 1983, ApJ, 264, 605

Miller Bertolami, M. M., \& Althaus, L. G. 2006, A\&A, 454, 845

Quirion, P.-O., Fontaine G., \& Brassard, P. 2004, ApJ, 610, 436

Rauch, T., Köppen, J., \& Werner, K. 1994, A\&A, 286, 543

Rauch, T., Dreizler, S., \& Wolff, B. 1998, A\&A, 338, 651

Saio, H. 1996, in Hydrogen-deficient stars, ed. C. S. Jeffery, \& U. Heber, ASP (San Francisco), 96, 361

Schönberner, D. 1979, A\&A, 79, 108

Stanghellini, L., Cox, A. N., \& Starrfield, S. 1991, ApJ, 383, 766

Starrfield, S., Cox, A. N., Hodson, S. W., \& Pesnell, W. D. 1983, ApJ, 268, L27

Starrfield, S., Cox, A. N., Kidman, R. B., \& Pesnell, W. D. 1984, ApJ, 281, 800 Starrfield, S., Cox, A. N., Kidman, R. B., \& Pesnell, W. D. 1985, ApJ, 293, L23 Werner, K. 2001, ApSS, 275, 27

Werner, K., \& Herwig, F. 2006, PASP, 118, 183 